\title{
COMPARACIÓN DE LA RESISTENCIA A LA FUERZA TRACCIONAL EN CORONAS METAL CERÁMICAS, MEDIANTE EL USO DE DOS PROTOCOLOS
}

\section{COMPARISON OF THE RESISTANCE TO TRACTION FORCE IN CERAMIC METAL CROWNS BY USING TWO PROTOCOLS}

\author{
Gallego-Caicedo Juan ${ }^{1 *}$, Izquierdo-Cevallos Cristian ${ }^{2}$, Garzón-Rayo Herney ${ }^{3}$ \\ 1 Odontólogo Universidad Santiago de Cali, Especialista en Rehabilitación Oral, Universidad del Valle. Colombia \\ ${ }^{2}$ Universidad Javeriana Cali, Especialista en Rehabilitación Oral.Colombia ${ }^{3}$ Universidad Javeriana Cali, Especialista en \\ Rehabilitación Oral, Universidad Militar Nueva Granada Fundación CIEO. Colombia \\ *khristian_cuenca@hotmail.com
}

\begin{abstract}
Resumen
OBJETIVO: Determinar la diferencia en la fuerza traccional necesaria para desalojar las coronas metal cerámica con collarless (hombro cerámico) sobre dientes naturales extraídos utilizando dos diferentes protocolos de preparación de sustrato dental y un mismo cemento resinoso autoadhesivo de curado dual. MATERIALES Y MÉTODOS: treinta y cinco dientes recién extraídos por indicaciones ortodonticas fueron almacenados mediante protocolos de conservación, de los cuales treinta fueron preparados por un mismo operador y cinco fueron maquinados en torno computarizado para recibir coronas metal cerámica collar less. La muestra fue distribuida de la siguiente manera: quince dientes para utilización de cementación con preparación del sustrato dental, quince dientes para la utilización de cementación sin preparación del sustrato dental y cinco dientes maquinados para la utilización de cementación sin preparación del sustrato dental. La muestra fue sometida en cada

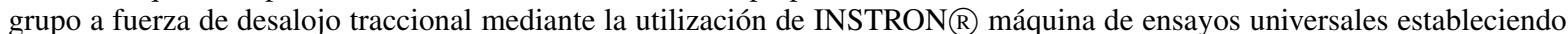
resultados para cada uno, se realizó comparación entre ellos y finalmente se determinó el tipo de falla en la cementación (adhesiva o cohesiva) para cada muestra. Se establecieron promedios y desviación estándar, el nivel de confianza se estableció en $95 \%$ y el nivel de significancia en $5 \%$. RESULTADOS: La medición de la fuerza de adhesión de las coronas permitió establecer que la resistencia a la tracción antes del desalojo si tuvo diferencias estadísticamente significativas $(\mathrm{p}<0.05)$ en al menos dos de los tres grupos, específicamente los grupos SP (control) y CP. Esto se obtuvo al aplicar la prueba no paramétrica de Kruskal-Wallis. CONCLUSIONES: Dentro de las limitaciones del presente estudio se puede concluir que las preparaciones cercanas a los 15 grados de convergencia oclusal que se obtienen al realizarlas de manera manual son aceptables para obtener una resistencia a la tracción adecuada de coronas metal cerámicas collarless cementadas con cemento RelyX U200 3M ESPE.
\end{abstract}

Palabras clave: Retención de coronas, coronas collarless preparación dental, cemento de resina autoadhesivo.

\begin{abstract}
AIM Determine the difference in the tensile force needed to dislodge the metal ceramic crowns with collarless (CERAMIC SHOULDER) on extracted natural teeth using two different protocols for preparing dental substrate and the same dual-cure adhesive resin cement. MATERIALS AND METHODS: Thirty-five recently extracted for orthodontic indications were stored by conservation protocols, of which thirty were prepared by the same operator and five teeth were machined on computerized lathe to receive metal ceramic collar less crowns. The sample was distributed as follows: fifteen teeth for use cementing preparation of dental substrate, fifteen teeth using cement without preparing the dental substrate and five teeth machined for using unprepared cementing dental substrate. The sample was subjected in each group to force tensile eviction using INSTRONR machine universal testing setting results for each comparison between them it was made and finally the type of failure was determined in the cement (adhesive or cohesive) to each sample. Means and standard deviations were established, the confidence level was set at 95\% and the significance level of 5\%. RESULTS: Measurement of adhesion strength of the crown established that the tensile strength before eviction if had statistically significant difference $(p<0.05)$ in at least two of the three groups, specifically the SP group (control) and CP. This is obtained by applying the nonparametric Kruskal-Wallis test. CONCLUSIONS: Within the limitations of this study it can be concluded that preparations near 15 degrees of cervical convergence obtained to perform manually are suitable for resistance adequate traction crowns metal ceramics collarless cemented with RelyX U200 3M ESPE.
\end{abstract}

Key words: Crown retention, collarless crowns, tooth preparation, self - adhesive resin cement. 


\section{INTRODUCCIÓN}

Las coronas metal cerámica en la actualidad aún son ampliamente usadas en la práctica odontológica y consideradas como el "gold standart" de las restauraciones de cubrimiento total, aún con el mejoramiento en el campo de las cerámicas libre de metal; las coronas metal cerámica collarless son restauraciones a la cual se les ha eliminado la porción cervical metálica y ha sido reemplazada por cerámica la cual está en contacto directo con la preparación dental en la zona de la línea terminal, ${ }^{1}$ son una opción terapéutica que brinda las ventajas de la resistencia del metal con el aspecto estético de la cerámica. ${ }^{2}$

Dentro de las diversas aleaciones disponibles en el mercado, las de uso más común son las aleaciones predominantemente base en especial aleaciones Ni-Cr (Níquel - Cromo) ya que además de su bajo costo y fácil técnica de fabricación poseen buenas propiedades. ${ }^{3}$

El diseño de una preparación para una restauración colada y su ejecución depende básicamente de cinco principios:

1) Preservación de la estructura dental,

2) Retención y resistencia,

3) Durabilidad estructural,

4) Integridad marginal

5) Preservación del periodonto.

Además de reemplazar la estructura dentaria perdida, una restauración debe preservar la estructura dentaria remanente. La configuración geométrica de la preparación dentaria debe situar al cemento bajo compresión con el fin de proporcionar la retención y la resistencia necesarias. La retención y la resistencia son dos cualidades que se interrelacionan y son inseparables. El elemento de la retención lo constituye dos superficies verticales opuestas en la misma preparación, estas pueden ser externas que se refieren específicamente al diente e internas que se refieren al interior de la restauración coronal. $^{1}$

Dado que una restauración colada o de metal se coloca sobre o en la preparación una vez que ha sido fabricada en su forma final, es importante que las paredes axiales de dicha preparación tengan una ligera conicidad que permita su colocación. Algunos autores como Shillingburg H.T. han propuesto que la configuración de las preparaciones para una corona completa idealmente sea de 6 grados de convergencia oclusal. Se encontró un promedio de 15.8 grados entre las paredes distal y mesial, y de 13.4 grados entre las paredes vestibular y lingual como medidas promedio en el momento de realizar las preparaciones. ${ }^{2}$ La configuración geométrica de un diente preparado es esencial para promover la retención entre las paredes axiales de la preparación y de la restauración protésica. ${ }^{3}$

La reducción del ángulo de convergencia, la altura de la superficie axial y una reducción mínima oclusal contribuyen a aumentar la capacidad de retención de un diente preparado. ${ }^{4}$
La restauración tipo corona metal cerámica fue descrita por primera vez por Brecker en 1956. ${ }^{1}$ Él describió el uso de un collar metálico circunferencial. El metal a nivel cervical sirve como una armadura que fortalece la fundición y permite la resistencia a la deformación durante los ciclos de cocción de la cerámica, pero produce una línea oscura o sombra debajo del tejido gingival. ${ }^{6}$ Así, los diseños alternativos a la estructura metálica se desarrollaron.

Se enfatizó en que el diseño de la subestructura metálica tenía una relación significativa a la resistencia de la ruptura final, lo que indicaba que conservar toda la estructura metálica era mejor que no tenerla. ${ }^{6}$ En otro estudio se encontró que reducir $1 \mathrm{~mm}$ de estructura metálica a nivel cervical contribuiría a disminuir el efecto de oscurecimiento producido por el metal lo cual no comprometería la resistencia a la fractura de la cerámica a este nivel. ${ }^{7}$ Realizar este procedimiento a nivel cervical mejora los resultados estéticos siempre y cuando se respeten los márgenes estipulados que garanticen la resistencia de la cerámica. ${ }^{8}$ Por lo tanto, se hicieron recomendaciones con respecto a los diseños de estructura que maximizan las características estéticas sin afectar la fuerza de restauraciones de cerámica y metal debido a la naturaleza frágil que presenta la cerámica, incluso si es reforzada puede conducir a la fractura, cuando se somete a fuerzas de cizalladura o de tensiones. ${ }^{9}$

La cementación de las restauraciones indirectas es considerada como la parte fundamental en cuanto al éxito a largo plazo. Dentro del espectro de los materiales dentales, los cementos resinosos, proporcionan una mejor estética, menor solubilidad y mejor adaptación marginal en comparación con los cementos tradicionales. ${ }^{10}$

Los cementos resinosos autoadhesivos son los que al momento, mayor desarrollo presentan. Se les ha conferido una técnica simplificada con menor cantidad de pasos clínicos en la preparación del sustrato dental que facilitan su uso, así como también refieren mejoradas características adhesivas y una amplia lista de indicaciones que les permite ser utilizados para cementar diferentes materiales con un mismo producto según las casas fabricantes. Es por esto que varios estudios se han realizado con el fin de compararlos entre sí, en determinadas situaciones experimentales se han realizado ensayos in vitro, en estudios comparativos entre medios húmedos y $\operatorname{secos}^{11} \mathrm{y}$ en situaciones de comportamiento a la carga cíclica a través del tiempo. ${ }^{12}$

En cuanto a su comportamiento en resistencia de cementación dependiendo de la humedad del sustrato dental al cual se van a adherir no se han encontrado diferencias significativas en diferentes grados de humedad y en seco, ni a las 24 horas de realizada la cementación, ni a los 6 meses. $^{11}$

En relación a la diferencia de acuerdo al ciclaje mecánico no se ha encontrado diferencias significativas entre grupos de prueba sometidos a ciclaje y grupos que no se sometieron al mismo. En cuanto a la degradación en saliva se encontró 
que no hubo diferencia estadísticamente significativa entre el grupo de prueba inmediata a la cementación y los grupos que se preservaron en saliva artificial por un periodo de uno y dos años. ${ }^{12}$

Con el transcurso del tiempo se ha evidenciado la duda de cementar una corona metal cerámica collarless con técnica convencional o técnica adhesiva, la información soportada por evidencia científica sobre este tema particular es escasa, por lo que con el fin de generar una claridad en cuanto a la retención obtenida según la técnica utilizada; surge la necesidad de realizar este estudio in vitro, como un primer paso para permitir aclarar si existe o no alguna ventaja de una técnica de preparación del sustrato dental sobre otra.

El propósito de este estudio es determinar la diferencia en la fuerza traccional necesaria para desalojar las coronas metal cerámica con collarless sobre dientes naturales extraídos utilizando dos diferentes protocolos de preparación de sustrato dental y un mismo cemento resinoso autoadhesivo de curado dual y determinar el tipo de falla (adhesiva o cohesiva) que se presentó en la prueba.

\section{MATERIALES Y MÉTODOS}

Se realizó un consentimiento informado que fué firmado por los pacientes con el fin de lograr la obtención de permisos para la utilización de dientes premolares inferiores extraídos por indicación de ortodoncia que cumplían con los criterios de inclusión: Dientes premolares humanos sanos o con restauraciones pequeñas en la superficie oclusal, que no tuvieran fracturas, endodoncia previa, alteraciones anatómicas de forma o tamaño en la zona de la corona, que tuvieran indicación para extracción por ortodoncia y que habían sido donados voluntariamente por los pacientes de acuerdo al consentimiento informado para este estudio. Una vez obtenidos los dientes para el estudio fueron lavados con agua no estéril para la eliminación de sangre y fluidos y se almacenaron en un recipiente con tapa, el cual contenía solución salina (Baxter TM ) a $37{ }^{\circ} \mathrm{C}$ con una humedad relativa del $100 \%$ y se cambió la solución salina cada dos semanas según las normas ICONTEC 4882/2000, e ISO/TS $11405 / 2003$ hasta recolectar toda la muestra necesaria que fueron cuarenta (40) dientes de los cuales cinco (5) dientes fueron utilizados en pruebas y ajustes y los 35 restantes para el estudio.

Un mismo operador insertó los dientes de manera centrada en tubos de PVC cilíndricos de veinticinco $(25 \mathrm{~mm})$ de alto por veinte $(20 \mathrm{~mm})$ de diámetro interno que contenían resina acrílica auto polimerizable (FTRA32-001 VERACRYL NEW STETIC SA) la resina acrílica se mantuvo a $3 \mathrm{~mm}$ por debajo de la línea amelo-cementaria. Una perforación horizontal se realizó en la raíz a una distancia equidistante entre la línea amelo-cementaria y el ápice para permitir el paso de un alambre circular de ortodoncia número ocho (8); y adicionalmente se realizó otra perforación a nivel más cervical de la raíz con el objetivo de aumentar la retención dentro del acrílico, a dicha perforación se le atravesó una broca de acero de 2 milímetros de diámetro.

Las preparaciones se realizaron utilizando un torno computarizado de control numérico (C.N.C. Computarizado Encoturn 240), asistido por el programa de diseño AUTOCAD, con el fin de tener preparaciones homogéneas y estandarizadas, de los cuales solo se obtuvieron 5 muestras debido a que el resto de dientes de prueba presentaron fracturas, descartando la realización total de las preparaciones dentales con este torno y obligando a realizar las 30 restantes de manera convencional por un mismo operador.(Figura 1)

Las preparaciones dentales fueron realizadas con las siguientes características tanto en el torno como por el operador: convergencia de las paredes axiales aproximadamente de 6 grados, altura del muñón mínima de $5 \mathrm{~mm}$ y línea de terminación tipo chanfer ${ }^{13}$ con una profundidad de $1,5 \mathrm{~mm}$.

Una vez preparados los dientes se les tomó una impresión a cada uno mediante el uso de un elastómero tipo polivinilsiloxano (Panasil Kettenbach), tipo masilla y liviana; el vaciado de estas impresiones se realizó con el fin de obtener troqueles en yeso dental tipo V (Jadestone - Whip Mix); la línea terminal se marcó con un lápiz de color rojo libre de carbón y grafito marca (Papermate colorchek ) y fueron despejados con el uso de pimpollos multilaminares para Micromotor por un mismo operador.

Los dientes preparados se dividieron en tres (3) grupos, dos de manera aleatoria: grupo de técnica adhesiva con preparación de sustrato dental al que se le domino $(\mathrm{CP})$, grupo de técnica adhesiva sin preparación de sustrato dental al que se le denomino (SP) y el tercero de técnica adhesiva sin preparación de sustrato dental en dientes maquinados al que se le denomino con la letra (M). Estos se numeraron e identificaron mediante un código alfanumérico donde se indica el grupo en letras y un número del 1 al 15 para los dos primeros grupos (CP) (SP) y del 1 al 5 para el último grupo $(\mathrm{M})$, marcándolos de forma continua.

\subsection{CONFECCIÓN DE LAS CORONAS}

Las cofias fueron enceradas sobre los troqueles de yeso obtenidos con anterioridad dejando $1.5 \mathrm{~mm}$ desde el margen de la preparación para el collar cerámico el cual fue elaborado con cerámica Margin IPS InLine Ivoclar Vivadent, y se revistieron con cerámica feldespática de recubrimiento IPS InLine Ivoclar Vivadent, el metal utilizado es una aleación de $\mathrm{Ni}-\mathrm{Cr}$ (Wiron Light, Bego) que es una aleación predominantemente base que contiene $64,5 \% \mathrm{Ni} ; 22 \% \mathrm{Cr} ; 10 \% \mathrm{Mo} ; 2,1 \mathrm{Si}$. Se probó la adaptación de las coronas en los dientes, mediante la utilización de estereomicroscopio, se terminaron las coronas con pulido y glaseado. 


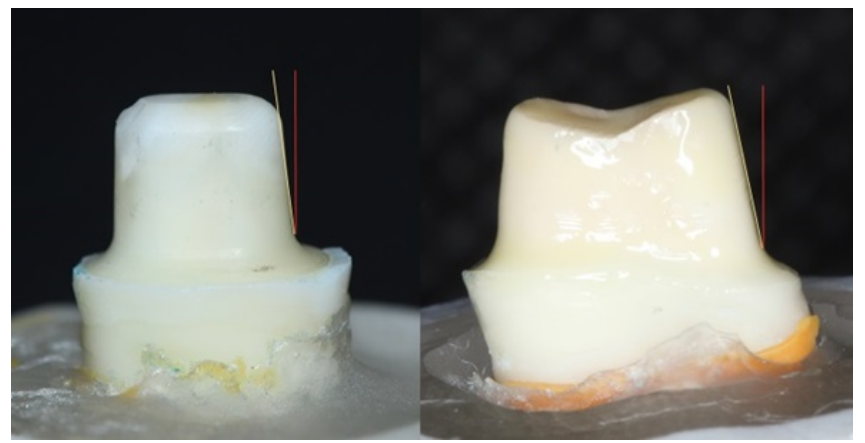

Fig. 1. Comparación de la angulación de las preparaciones entre (A) grupo $\mathrm{M}$ maquinadas se observa la inclinación de 3 grados de la pared en relación al plano vertical y (B) grupo SP preparadas a mano por un operador donde el ángulo de la preparación es cercano a los 7 grados en relación al eje vertical.

\subsection{LIMPIEZA DE LOS DIENTES PARA LA CEMENTA- CIÓN}

Las preparaciones se limpiaron mediante el uso de un cepillo rotatorio montado en una pieza de mano de baja velocidad (NSK ) y mezcla de agua con bicarbonato de sodio, la superficie se lavó con agua no estéril y secado con algodón.

\subsection{ACONDICIONAMIENTO DE LAS CORONAS}

$\mathrm{El}$ acondicionamiento de las coronas se realizó de igual manera para los tres grupos tanto CP, SP y M de la siguiente manera: La porción metálica de la corona fue arenada con óxido de aluminio en partículas de 50 micras,${ }^{14}$ limpiadas con alcohol y agua, secadas con chorro de aire libre de agua y aceite. El collar cerámico fue acondicionado con ácido hidrofluorhídrico al $9.6 \%$ (Eufar ) por 60 segundos, se lavó con mezcla de agua destilada y bicarbonato de sodio por 1 minuto, fueron llevadas a ultrasonido por 10 minutos y secadas con chorro de aire. ${ }^{15}$ Posteriormente se aplicó a repetición por 2 ocasiones silano (Ceramic Primer 3M ESPE ) sobre la superficie por 1 minuto cada una.

\subsection{ACONDICIONAMIENTO DE LAS CORONAS}

Grupo CP: Sobre la superficie dental previamente limpia $^{16}$ se colocó el adhesivo resinoso Single Bond Universal (3M ESPE ) el cual se mezcló por 5 segundos en una proporción de 1:1 con el activador de curado dual (DCA 3M ESPE ), se aplicó en el diente mediante el uso de un aplicador desechable microbrush frotándolo por 10 segundos siguiendo las indicaciones del fabricante. Grupo SP: Ninguna preparación se realizó sobre el sustrato dental una vez terminada la limpieza y secado de la misma. Grupo M: Igual que el grupo SP.

\subsection{CEMENTACIÓN}

Se dispensó el cemento resinoso RelyX U200 Automix (3M - ESPE ) mediante el uso del sistema automix y se colocó en la zona del collar cerámico, se llevó la corona al diente y se presionó digitalmente, luego se sometió a una presión constante de $5 \mathrm{~kg}$ por 10 minutos en una prensa personalizada para este fin. ${ }^{17} \mathrm{El}$ exceso de material se retiró con un explorador después de un fotocurado inicial de 2 segundos con la lámpara de fotocurado, con el fin de facilitar el retiro de los excesos, luego de esto se realizó el fotocurado final de la superficie cerámica del collarless 40 segundos a la distancia establecida mediante el contacto de la punta siliconada de la lámpara.

\subsection{ALMACENAMIENTO DE LOS ESPECÍMENES}

Una vez cementadas las coronas los especímenes fueron almacenados en agua destilada a temperatura ambiente previo al inicio de las pruebas de resistencia a la fuerza traccional.

\subsection{PRUEBA DE FUERZA TRACCIONAL}

La retención de las coronas fue medida aplicando una fuerza traccional al anillo adherido a la porción oclusal de cada corona en una máquina de ensayos universales (INSTRON 5686) del laboratorio de solidos de la Universidad Autónoma de Occidente Cali, a una velocidad de $0.5 \mathrm{~mm} / \mathrm{min}$. Cada espécimen se colocó en la parte inferior de la máquina, la parte superior de la misma tenía un pasador de acero de 2.5 $\mathrm{mm}$ de diámetro que atravesaba de lado a lado el anillo de cada corona. Durante este proceso se perdieron por motivos accidentales 1 muestra de cada grupo, dejando en total 32 muestras para el estudio. Los valores de fuerza traccional necesarios para desalojar la corona fueron registrados en un Quimografo (INSTRON 5686), el cual es el sistema computarizado de la máquina de ensayos universales INSTRON expresados en Megapascales (MPa). Posteriormente se observó cada corona y diente para determinar qué tipo de falla hubo en la cementación (adhesiva o cohesiva)

\section{RESULTADOS}

Fueron evaluadas 32 muestras de dientes con coronas cementadas en tres grupos experimentales $(\mathrm{M}=4, \mathrm{SP}=14$, $\mathrm{CP}=14)$. La medición de la fuerza de adhesión de las coronas permitió establecer que la fuerza promedio de resistencia a la tracción antes del desalojo fue de 453.75 con una variante de 183.58 MPa en el grupo M, mientras que para los grupos SP y CP fue de 29.35 con una variante de $26.673 \mathrm{MPa}$ y 350.57 con una variante de $121.99 \mathrm{MPa}$ respectivamente.

Los valores de fuerza en el grupo $\mathrm{M}$ fueron mayores a 400 MPa para más del $50 \%$ de la muestra, mientras que para CP más del $50 \%$ se encontró por debajo de esta cifra, y para SP el $100 \%$ no alcanzo los $100 \mathrm{MPa}$ (Figura 2). Mediante contraste de hipótesis al aplicar la prueba no paramétrica de Kruskal-Wallis se logra identificar que las diferencias son estadísticamente significativas en al menos dos de los grupos (figura 2). Las diferencias se encontraron entre los grupos SP y CP (figura 5) 


\begin{tabular}{|c|c|c|}
\hline Grupo 1 ExpeI & Grupo 2 Control & Grupo 3 Experimental \\
\hline $\begin{array}{l}\text { - Unidad experimental: } \\
\text { un diente premolar con } \\
\text { corona collar less. } \\
\text { - } \mathrm{n} \text { : } 15 \\
\text { - Experimento: } \\
\text { Cementación con } \\
\text { protocolo adhesivo con } \\
\text { preparación } \\
\text { sustrato dental. } \\
\text { Información: Valor } \\
\text { máximo registrado } \\
\text { para la } \\
\text { descementación de la } \\
\text { corona del diente } \\
\text { mediante fuerza } \\
\text { traccional. }\end{array}$ & $\begin{array}{l}\text { - Unidad experimental: } \\
\text { un diente premolar } \\
\text { con corona collar } \\
\text { less. } \\
\text { - n: } 15 \\
\text { - Experimento: } \\
\text { Cementación con } \\
\text { protocolo adhesivo } \\
\text { sin preparación del } \\
\text { sustrato dental. } \\
\text { Información: Valor } \\
\text { máximo registrado } \\
\text { para la } \\
\text { descementación de } \\
\text { la corona del diente } \\
\text { mediante fuerza } \\
\text { traccional. }\end{array}$ & $\begin{array}{l}\text { - Unidad } \\
\text { experimental: un } \\
\text { diente premolar } \\
\text { maquinado con } \\
\text { corona collar less } \\
\text { - n: } 5 \\
\text { - Experimento: } \\
\text { cementación con } \\
\text { protocolo adhesivo } \\
\text { sin preparación del } \\
\text { sustrato dental. } \\
\text { Información: valor } \\
\text { máximo registrado } \\
\text { para la } \\
\text { descementación de } \\
\text { la corona del diente } \\
\text { mediante fuerza } \\
\text { traccional. }\end{array}$ \\
\hline
\end{tabular}

Tabla 1. Grupos de Estudio

\begin{tabular}{cccc}
\hline Grupo & N & $\begin{array}{c}\text { Promedio (MPa) } \pm \\
\text { Desviación Estándar }\end{array}$ & P \\
\hline$M$ & 4 & $45.375+18.35854$ & \\
$\mathrm{SP}$ & 14 & $29.35+2667.355$ & 0.0001 \\
$\mathrm{CP}$ & 14 & $350.57 \pm 121.996$ & \\
\hline
\end{tabular}

Tabla 2. Grupos de valores de la fuerzas 


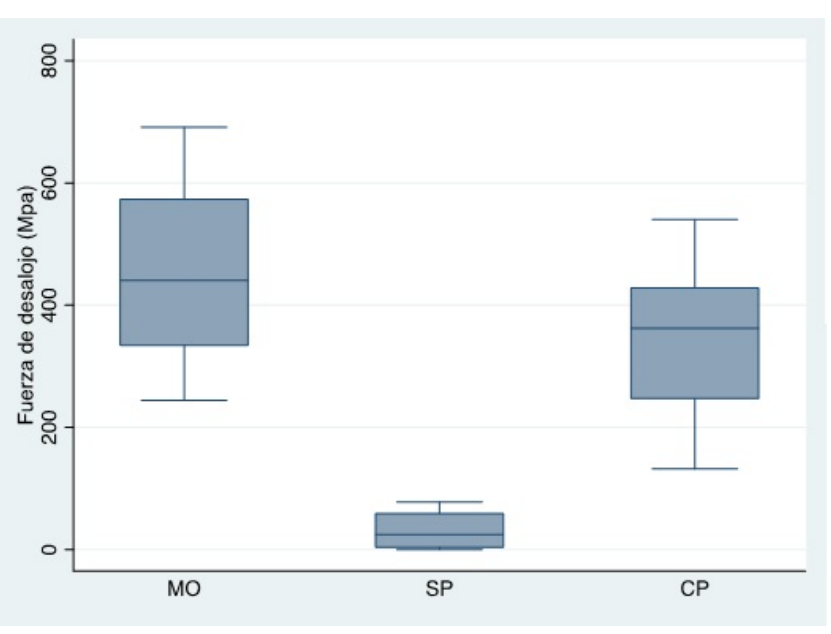

Fig. 2. Diferencia entre los grupos SP y CP

\section{DISCUSIÓN}

El resultado de este estudio en relación a la convergencia de las paredes de la preparación mostró que en los dientes preparados de manera manual se obtuvieron preparaciones de aproximadamente 15 grados de convergencia similar a lo descrito por Goodacre C.J. 1977, ${ }^{8}$ mientras que en las que se realizaron de manera maquinada se obtuvieron convergencias de 6 grados como las sugeridas por Potts R.G. y Col. 1980. ${ }^{5}$ En cuanto a la resistencia al desalojo durante las pruebas traccionales, en el presente estudio se encontraron valores de aproximadamente $453.75 \mathrm{MPa}$ en el grupo de dientes maquinados y cementados con cemento resinoso RelyX U200 3M ESPE, lo que difiere a los resultados encontrados en estudios como el de Marchiori M. 2010, ${ }^{17}$ donde en una prueba de tracción de coronas realizadas sobre dientes maquinados en un torno, los valores encontrados de resistencia a la tracción fueron aproximadamente de $3,887 \mathrm{MPa}$, estas coronas fueron cementadas con cemento resinoso autoadhesivo Relyx U100 3M ESPE. En otro estudio donde también se cementaron coronas sobre dientes preparados mediante un torno sobre los cuales se elaboraron coronas metálicas que fueron sometidas a tracción realizado por Mohamed F. y Col. $2009^{18}$ se obtuvieron resultados de 7.4 MPa en el grupo que utilizó un cemento resinoso de curado dual Panavia 21 Kuraray. En el grupo de dientes que se prepararon manualmente por un operador en el presente estudio se encontraron valores de aproximadamente 29.35 MPa, para el grupo en el que se utilizó el protocolo sugerido por la casa comercial del cemento, encontrando valores diferentes a los encontrados por Veerabadhran M. M. $2012^{19}$ donde los valores más altos obtenidos fueron de 1.89MPa, utilizando un cemento de ionomero de vidrio modificado con resina FujiCem GC América y de 7.4 MPa en los resultados obtenidos por Mohamed y Col. $2009^{18}$ utilizando Panavia 21 Kuraray en el grupo de dientes q se maquinaron a 12 grados de convergencia de la preparación.
Por otro lado, si comparamos los valores obtenidos dentro del presente estudio con los valores suministrados por la casa comercial para la retención a la fuerza traccional sobre sustrato dental utilizando cemento resinoso RelyX U200 $3 \mathrm{M}$ ESPE que es de aproximadamente $25 \mathrm{MPa},{ }^{20}$ podemos encontrar que los resultados son similares en cuanto a la fuerza necesaria para desalojar las coronas. En el grupo que se utilizó un protocolo experimental en este estudio los valores de resistencia a la tracción fueron aproximadamente de $350 \mathrm{MPa}$ en el grupo de dientes preparados manualmente, es decir con una angulación cercana a los 15 grados, lo cual es diferente a los valores encontrados en estudios como el de Mohamed y Col. 2009, ${ }^{18}$ en donde utilizaron angulaciones similares por lo que podemos suponer que el cambio del protocolo de preparación del sustrato empleado en el grupo $\mathrm{CP}$ de este estudio puede ser el responsable del aumento de la resistencia traccional para este caso. En el presente estudio se utilizó un cemento resinoso de curado dual autoadhesivo y auto grabador, donde se obtuvieron valores de resistencia a la tracción de $450 \mathrm{MPa}$ siguiendo el protocolo de la casa comercial, valores similares de 239. $22 \mathrm{MPa}$ que obtuvieron Amarnath G S. y Col. $2015^{21}$ en el grupo de muestras similares a las del presente estudio utilizando un cemento resinoso Unicem 3M ESPE.

En cuanto a los valores de resistencia a la tracción de la dentina de $44.4 \mathrm{MPa}$ cerca de la pulpa según reportan estudios como el de Staninec M. $2002^{22}$ y 39 MPa reportados por Smith D.C. $1971^{23}$ contrastan con los valores encontrados durante las pruebas de tracción del presente estudio donde las fallas cohesivas del sustrato dental se presentaron en las muestras que superaron los $400 \mathrm{MPa}$ de retención a la tracción aproximadamente, las demás muestras que presentaron valores de retención a la tracción inferiores a los $400 \mathrm{MPa}$ presentaron fallas adhesivas del cemento al sustrato dental y no existieron fallas adhesivas del cemento a la corona. Tomando en cuenta los valores de resistencia traccional de las fibras de colágeno de la capa hibrida que es de $25 \mathrm{MPa}$ según reportan Kinney J. $2003^{24}$ y $29 \mathrm{MPa}$ reportado por Sano H. $1994,{ }^{25}$ y la resistencia traccional de la dentina que esta alrededor de los $40 \mathrm{MPa}$ podremos determinar que el valor mínimo requerido de resistencia traccional en un cemento deberá estar cerca de estos valores por lo cual encontramos que todos los protocolos que se utilizaron en el presente estudio son adecuados para la cementación definitiva de coronas metal cerámicas collarless.

\section{CONCLUSIONES}

Dentro de las limitaciones del presente estudio, se puede concluir que las preparaciones cercanas a los 15 grados de convergencia oclusal que se obtuvieron al ser realizadas de manera manual son aceptables para obtener una resistencia a la tracción adecuada de coronas metal cerámicas collarless cementadas con cemento de resina RelyX U200 3M ESPE. 


\section{Comparaciones múltiples}

Variable dependiente: Fuerza

Tamaño

\begin{tabular}{|c|c|c|c|c|c|c|}
\hline \multirow[b]{2}{*}{ (I) Grupo } & \multirow[b]{2}{*}{ (J) Grupo } & \multirow{2}{*}{$\begin{array}{c}\text { Diferencia de } \\
\text { medias (I-J) }\end{array}$} & \multirow[b]{2}{*}{ Error estándar } & \multirow[b]{2}{*}{ Sig. } & \multicolumn{2}{|c|}{$95 \%$ de intervalo de confianza } \\
\hline & & & & & Límite inferior & Límite superior \\
\hline \multirow[t]{2}{*}{ M } & SP & 424,393 & 92,069 &, 055 & $-15,52$ & 864,31 \\
\hline & $\mathrm{CP}$ & 103,179 & 97,411 & ,728 & $-293,31$ & 499,67 \\
\hline \multirow[t]{2}{*}{ SP } & M & $-424,393$ & 92,069 & 055 & $-864,31$ & 15,52 \\
\hline & $\mathrm{CP}$ & $-321,214^{*}$ & 33,375 & .000 & -411.43 & $-231,00$ \\
\hline \multirow[t]{2}{*}{$\mathrm{CP}$} & M & $-103,179$ & 97,411 & ,728 & $-499,67$ & 293,31 \\
\hline & $\mathrm{SP}$ & $321,214^{*}$ & 33,375 & .000 & 231,00 & 411,43 \\
\hline
\end{tabular}

*. La diferencia de medias es significativa en el nivel 0.05 .

Fig. 3. Diferencias de medidas

También se puede concluir que todos los protocolos adhesivos que se utilizaron en el presente estudio, aunque presentaron diferencias estadísticamente significativas en sus valores de resistencia a la tracción, fueron adecuados para generar valores de retención necesarios en las coronas metal cerámica collarless al compararlos con los valores que muestra la evidencia científica. Con las limitaciones del presente estudio in vitro, podemos sugerir el uso de un protocolo de preparación de sustrato dental con adhesivo igual al realizado en el grupo $\mathrm{CP}$ en preparaciones muy cónicas, con el fin de aumentar la retención que se encuentra disminuida por la expulsividad de la preparación.

Conflicto de intereses y financiamiento Los autores declaran no tener conflicto de intereses, haber cumplido con los requisitos de autoría y haber autofinanciado este artículo.

\section{Referencias}

1 The Academy of Prostodonthics. The glossary of prosthodontic terms. Journal Prosthetic Dentistry. July 2005.Vo194.Num1.

2 Sikka Swati. Marginal Strength of collarless Metal Ceramic Crown Int. Journal of Dentistry. Vol.2010 AID.521470

3 Huang $\mathrm{HH}$. Effect of chemical composition of $\mathrm{Ni}-\mathrm{Cr}$ dental casting alloy on the bonding characterization between porcelain and metal. Journal Of Oral Rehabilitation 2005.32;2006-212.
4 Shillingburg HT, Hobo S, Whitsett LD. Fundamentos de protesis fija. Prosthodontics. 3nd edicion. Chicago: Quintessence Books .2001.

5 Potts RG, Shillingburg HT Jr, Duncanson MG Jr. Retention and resistance of preparations for cast restorations. J Prosthet Dent. 1980; 43: 303-8.

6 Ayad MF, Johnston WM, Rosenstiel SF. Influence of tooth preparation taper and cement type on recementation strength of complete metal crowns. J Prosthet Dent. 2009; 102: 354-61.

7 Brecker SC. Porcelain baked to gold a new medium in prosthodontics. J Prosthet Dent 1956;6:801-10.

8 Goodacre CJ, Van Roekel NB, Dykema RW, Ullmann RB. The collarless metal-ceramic crown. J Prosthet Dent 1977;38: 615-22.

9 Warpeha WS Jr, Goodkind RJ. Design and technique variable affecting fracture resistance of metal-ceramic restorations.J Prosthet Dent 1976;35:291-8.

10 Susan Hattar. Shear Bond strength of self-adhesive resin cements to base metal alloy. Journal of Prosthetic Dentistry. Article in press.

11 Carolina Bosso Anré. Tahiane R.A. Bond strength of self adhesive to dry and moist dentin. Braz Oral Restoration. 2013 Sep-Oct; 27(5): 389-05.

12 Tahiane R.A. Carolina B.A. Effect of storage times and mechanical load cycling on dentin bond strength of conventional and self-adhesive resin luting cement. Journal of Prosthetic Dentistry, Article in press. O'Boyle KH, Norling BK, Cagna DR, Phoenix RD. An investigation of new metal 
framework design for metal ceramic restorations. J Prosthet Dent 1997;78:295-301.

13 Satterthwaite, J. Indirect restoration on theet with reduced Crown heigth. Dental Update, V33, Pag 210-216, 2006.

14 Veerabadhran MM. The effect of retentive grove sandblsting and cement type on the retentive strenght of stainless Steel Crown in primary secound molars- An in vitro compartative study Jour. Indian Soc. of pedodontics and preventive dentistry Juan- March 2012 Issues 1 Vol. 30

15 Borges Gilberto. Effect of etching and airborne particle abrasion on the microstructure of different dental ceramics. J Prosthet Dent 2003; 89:479-88.

16 Chaiyabutr Y. The effects of tooth preparations cleansing protocols on the bond strenght of self-adhesive resin luting cement to contaminated dentin Operative Dentistry 2008, 33-5, 556-563.

17 Marchiori M. Influence of preparation height and luting agent type on Crown retention in molars. Braz. Jour. Sci. Apl-Jun. 2010. Vol 9 Issues 2.

18 Mohamed F. A. Influence of tooth preparation taper and cement type on recementation strenght of complete metal crowns. The journal of Prosthetic Dentistry 2009;102:354361.

19 Veerabadahran M.N. The effect of retentive Groove sand blasting annd cement type on the retentive strenght of stainless Steel crowns in primary second molars An In Vitro comparative study. Journal of indian Society of Pedodontics and preventive dentistry.Jan- Mar 2012 Issue 1 Vol 30. 1926.

20 3M ESPE. Technical data sheet. RelyX U200 Self adhesive resin cement. 2011.

21 Amarnatn G. S, et Al. Comparative evaluation of enhancing retention of dislogued crowns using preparation modification and luting cements: An in Vitro Study. Journal Of International Oral Health 2015;7(8):47-51.

22 Staninec M, et Al. Ultimate tennsile strenght of dentin: evidence for a damage mechanichs aproach to dentin failure. Journal of Biomedical Materials Research(APPL BIO MAT) 63:342-345,2002.

23 Smith D.C, et Al. The determination of shear strength. A metod using a micro punch apparatus. British Dental Journal.1971;130:333-337.

24 Kinney J. H. The mechanical propertyes of human dentin: A critical review and evaluation of dental literatura. Critical Review Biological Medical 14(19):13-29(2003).

25 Sano H. Tensil propertyes of mineralized and demineralized human and bovine dentin. Journal Dental Research 1994;73:1205-1211.

Recibido: 18 de Julio de 2016

Aceptado: 05 de Agosto de 2016 